\title{
The blood fluke Schistosoma mansoni cleaves the coagulation protein high molecular weight kininogen (HK) but does not generate the vasodilator bradykinin
}

\author{
Qiang Wang ${ }^{*}$ D, Akram A. Da'dara and Patrick J. Skelly
}

\begin{abstract}
Background: Schistosomes are blood dwelling parasitic worms that cause the debilitating disease schistosomiasis. Here we examined the influence of the parasites on their external environment by monitoring the impact of adult Schistosoma mansoni worms on the murine plasma proteome in vitro and, in particular, on how the worms affect the blood coagulation protein high molecular weight kininogen (HK).

Methods: Following the incubation of adult schistosomes in murine plasma, two-dimensional differential in-gel electrophoresis (2D-DIGE) was conducted to look for changes in the plasma proteome compared with control plasma. A major change to the blood protein kininogen (HK) was observed, and the interaction of Schistosoma mansoni parasite with this protein alone was then investigated by western blot analysis and activity assays. Finally, the generation of bradykinin from HK was monitored using a bradykinin detection kit.

Results: The most striking change to the plasma proteome concerned HK; while the full-length protein was more abundant in control plasma, carboxyl-terminal truncated forms were more abundant in plasma that contained schistosomes. Incubating parasites in buffer with pure HK followed by Western blot analysis confirmed that human HK is degraded by the worms. The resulting digestion pattern differed from that brought about by kallikrein, a host serine protease that normally acts on HK to release the vasodilator bradykinin. We found that live schistosomes, while digesting HK, do not generate bradykinin nor do they cleave a chromogenic kallikrein substrate. Since the cleavage of HK by the worms is not impeded by the serine protease inhibitor PMSF but is blocked by the cysteine protease inhibitor E64c, we hypothesize that schistosome tegumental cysteine proteases are responsible for HK cleavage.

Conclusions: Since proteomic and biochemical studies have revealed that the schistosome tegument contains two cysteine proteases belonging to the calpain family (SmCalp1 and SmCalp2) we conclude that these are likely responsible for the HK cleavage reported here. Schistosome cleavage of HK should help impede blood clotting and inflammation around the worms in vivo and so promote their ease of movement within the vasculature of their hosts.
\end{abstract}

Keywords: Schistosoma mansoni, Coagulation, High molecular weight kininogen, Bradykinin, Calpain

\footnotetext{
* Correspondence: Qiang.Wang@Tufts.edu

Molecular Helminthology Laboratory, Department of Infectious Disease and

Global Health, Cummings School of Veterinary Medicine, Tufts University,

North Grafton, MA, USA
} 


\section{Background}

Schistosomes are parasitic flatworms that live in the vascular system of vertebrates, causing a chronic, debilitating disease called schistosomiasis. Over 200 million people are estimated to be infected with schistosomes in over 70 countries [1] with another 800 million people at risk of infection [2]. Annual mortality in sub-Saharan Africa alone is over 250,000 people, with many more experiencing chronic morbidity [3, 4]. Three major species infect humans; these are Schistosoma mansoni, S. japonicum and S. haematobium [5]. Schistosomiasis is a water-borne disease, and infection occurs when freeswimming larval parasites called cercariae penetrate the skin. Here, each cercaria transforms into a morphologically and biochemically distinct life stage called the schistosomulum. Schistosomula invade a blood vessel, migrate through the heart and lungs to the liver where the worms mature and males pair with females. S. mansoni and $S$. japonicum couples then travel to the mesenteric veins, while $S$. haematobium pairs migrate to the vesicle venous plexus, where egg-laying occurs [6].

We are interested in how schistosomes modify their environment to promote their survival, and we are investigating the molecular capabilities of selected proteins expressed at the worm surface [7-10]. Here, we explored the impact of adult schistosomes on the murine plasma proteome. After incubating schistosomes in plasma, the most dramatic change seen in the plasma proteome is cleavage of high molecular weight kininogen (HK). HK is a circulating blood protein that is involved in the early stages of what is called the intrinsic coagulation pathway. HK is not enzymatically active; it functions as a cofactor in the conversion of coagulation factor XII to its active form (factor XIIa) and the conversion of prekallikrein to its active form (kallikrein) [11]. HK is also necessary for the activation of coagulation factor XI by factor XIIa [11]. In addition to acting as a cofactor, HK can itself be acted upon by kallikrein to generate the 9-amino acid inflammatory mediator bradykinin [12].

It has been reported that schistosome homogenates do possess a kallikrein-like activity, associated with an uncharacterized $66 \mathrm{kDa}$ protein (designated sK1) that can hydrolyze the kallikrein synthetic substrate d-ProPhe-Arg-p-nitroanilide [13]. The sK1 protein is localized to the tegumental surface of the adult parasite [13]. Also, an $S$. mansoni cDNA encoding a mouse plasma kallikrein homolog was identified (designated SmSP1) [14]. Since this cloned DNA encodes a $\sim 35 \mathrm{kDa}$ protein, this molecule differs from the larger, sK1 protein. SmSP1 was detected in schistosomula released products and male dorsal spines [14]. These host-exposed localization sites of sK1 and SmSP1 are consistent with schistosomes possessing a kallikrein-like activity.
Here, we monitor the ability of schistosomes to cleave HK ex vivo and compare this to the action of the host HK cleaving enzyme kallikrein. Additionally, we look for the ability of the worms to generate the vasodilator bradykinin. This work is designed to understand better the molecular capabilities of schistosomes that permit them to survive in the vasculature of their hosts for many years.

\section{Methods}

\section{Parasites and mice}

Schistosoma mansoni infected Biomphalaria glabrata snails (strain NMRI) were obtained from the Schistosomiasis Resource Center at the Biomedical Research Institute (BRI), Rockville MD. Larval schistosomes (cercariae, strain NMRI) were obtained from the infected snails, and schistosomula were prepared as described [15]. Adult male and female parasites were recovered by perfusion from Swiss Webster mice that were infected with $120 \mathrm{~S}$. mansoni cercariae seven weeks previously. All parasites were cultured in complete Dulbecco's Modified Eagle Medium/Nutrient Mixture F-12 (DMEM/F12) medium supplemented with $10 \%$ heat-inactivated fetal bovine serum, $200 \mathrm{U} / \mathrm{ml}$ penicillin and $200 \mu \mathrm{g} / \mathrm{ml}$ streptomycin, $0.2 \mu \mathrm{M}$ Triiodo-lthyronine, $1 \mu \mathrm{M}$ serotonin and $8 \mu \mathrm{g} / \mathrm{ml}$ human insulin and were maintained at $37{ }^{\circ} \mathrm{C}$, in an atmosphere of $5 \%$ $\mathrm{CO}_{2}$ [16]. All protocols involving animals were approved by the Institutional Animal Care and Use Committees (IACUC) of Tufts University under protocol G2015-113.

\section{Treatment of mouse plasma with schistosome parasites}

To obtain plasma, mouse blood was collected from the tale veins of 10 Swiss Webster mice into a collecting tube containing heparin. Samples were pooled, centrifuged at $13,000 \times \mathrm{rpm}$ for $15 \mathrm{~min}$ at $4{ }^{\circ} \mathrm{C}$, and the supernatant (plasma) recovered. Adult parasites were recovered from the culture medium and washed with Roswell Park Memorial Institute (RPMI) medium five times. Approximately 50 pairs of adult worms were incubated in $250 \mu \mathrm{l}$ of heparinized mouse plasma with $2 \mathrm{mM} \mathrm{CaCl}_{2}$ for one hour at $37{ }^{\circ} \mathrm{C}$. As a control, heparinized mouse plasma alone with $2 \mathrm{mM} \mathrm{CaCl}_{2}$ was likewise incubated at $37{ }^{\circ} \mathrm{C}$ for $1 \mathrm{~h}$. After that, parasites were removed, and plasma samples were submitted to Applied Biomics Inc. (Hayward, CA, USA) for two-dimensional differential ingel electrophoresis (2D-DIGE) and proteomic analysis.

\section{Two-dimensional differential in-gel electrophoresis (2D-DIGE)}

At Applied Biomics Inc., equal plasma samples were labelled with CyDye fluors: Cy2 (for the control (no parasite) sample) or Cy3 (for the plasma sample incubated with parasites for $1 \mathrm{~h}$ ). Labeled samples were then mixed with rehydration buffer, and proteins were 
resolved first by isoelectric focusing and then by sodium dodecyl sulfate-polyacrylamide gel electrophoresis (SDSPAGE) in the second dimension. The resulting 2-D gel was scanned using a Typhoon image scanner to reveal each of the CyDye signals (Cy2 and $\mathrm{Cy} 3)$. The scanned image was analyzed using ImageQuant software and comparative analysis of all spots was performed using DeCyder "in-gel" analysis software (Applied Biomics) to generate protein level ratios. To determine the identities of the selected proteins within the gel, spots of interest were picked using an Ettan Spot Picker. Excised spots were subjected to in-gel trypsin digestion followed by mass spectrometry (MS) analysis at Applied Biomics, Inc. Protein identification was based on peptide fingerprint mass mapping (using MS spectra) and peptide fragmentation mapping (using MS/MS spectra). Combined MS and MS/MS spectra were subjected to database searching using GPS Explorer software equipped with the MASCOT search engine to identify proteins from primary murine and schistosome sequence databases.

\section{High molecular weight kininogen (HK) assay}

Schistosomula $(\sim 1000)$ or adult parasites (5) were washed with Hanks balanced salt solution (HBSS) 3 times, and then resuspended in $150 \mu \mathrm{l}$ assay buffer (20 $\mathrm{mM}$ HEPES, $130 \mathrm{mM} \mathrm{NaCl}, 1 \mathrm{mM}$ EDTA, $10 \mathrm{mM}$ Glucose). An equal volume $(150 \mu \mathrm{l})$ of $6 \mathrm{mM} \mathrm{CaCl}_{2}, 10$ $\mathrm{mM} 2$-mercaptoethanol and $20 \mathrm{ng} / \mu \mathrm{l}(180 \mathrm{nM})$ plasmapurified, human HK (HK-HMW, Molecular Innovations, USA) was then added to each well. Next, parasites were incubated at $37{ }^{\circ} \mathrm{C}$ for $1 \mathrm{~h}$. Controls include the same HK solution to which $1 \mu \mathrm{g}$ of plasma-purified, human kallikrein (Millipore Sigma, Burlington, USA) was added or the HK solution without parasites. In some experiments, the serine protease inhibitor phenylmethylsulfonyl fluoride (PMSF, $0.5 \mathrm{mM}$, Sigma-Aldrich, St. Louis, USA) or the cysteine protease inhibitor E64c (0.1 mM, E0514, Sigma-Aldrich) was also added to the HK solution containing parasites. At selected time points (routinely, at $6 \mathrm{~h}$ and $24 \mathrm{~h}$ ) $30 \mu \mathrm{l}$ aliquots were recovered from each treatment group and analyzed by western blotting and for bradykinin generation.

\section{SDS-PAGE and Western blot analysis}

Samples recovered from the HK assay described above were resolved by 4-20\% SDS-PAGE (BioRad, Hercules, USA) as previously described [17]. Proteins were then transferred to PVDF membrane and blocked with TBST (Tris-buffered saline, $\mathrm{pH} 7.5,0.05 \%$ Tween 20) containing $5 \%$ dry non-fat milk powder for $1 \mathrm{~h}$ at room temperature. The membrane was then incubated with primary anti-HK antibody (Abcam, Cambridge, United Kingdom, 1:1000 dilution) for $1 \mathrm{~h}$ at room temperature, followed by washing with TBST buffer for $30 \mathrm{~min}$ and incubation with goat anti-rabbit IgG conjugated to horseradish peroxidase (GE Healthcare, Marlborough, USA) at 1:5000, for $1 \mathrm{~h}$ at room temperature. The blots were developed using Amersham ECL Detection Reagents (GE Healthcare) according to the manufacturer's instructions and images were recorded using a ChemiDoc ${ }^{\mathrm{TM}}$ Imaging System (Bio-Rad).

\section{Kallikrein assay}

Schistosomula $(\sim 1000)$ or adult parasites (5) were washed with HBSS 3 times, resuspended in $100 \mu \mathrm{l}$ assay buffer (described above) and placed into a well of a 48-well tissue culture plate. An equal volume $(100 \mu \mathrm{l})$ of $6 \mathrm{mM} \mathrm{CaCl}_{2}, 10 \mathrm{mM}$ 2-mercaptoethanol, with or without $1 \mathrm{mM}$ PMSF and $800 \mu \mathrm{M}$ kallikrein substrate (D-Pro-Phe-Arg-pNa, CS-31(02), HYPHEN Biomed, Neuville-sur-Oise, France) was added into each well. The product generated (para-nitro-aniline (pNA)) was monitored at OD $405 \mathrm{~nm}$ over time at $37{ }^{\circ} \mathrm{C}$. Control wells contained the kallikrein substrate solution without parasites (negative control) and the kallikrein solution containing plasma purified, human kallikrein (0.02 $\mu \mathrm{g}$, positive control, Millipore Sigma).

\section{Bradykinin measurement}

Samples recovered at $6 \mathrm{~h}$ and $24 \mathrm{~h}$ in the HK assay (described above) were analyzed for the presence of the 9-amino acid HK cleavage product bradykinin using a Bradykinin ELISA kit, (Abcam) and following the manufacturer's protocol. In short, bradykinin standards and samples were prepared and added to a series of wells of a capture antibody-coated 96-well plate. Then a biotinylated bradykinin conjugate was added to each well, followed by an anti-bradykinin polyclonal antibody. The mixture was then incubated at room temperature for 2 h. The plate was then washed 5 times using the wash buffer provided, and a streptavidin-HRP solution was added to all wells. Next, the plate was incubated for 30 min, washed a further 5 times before tetramethylbenzidine (TMB) substrate solution was added to all wells. After room temperature incubation for $30 \mathrm{~min}$, stop solution was added and the plate was read at OD $450 \mathrm{~nm}$.

\section{Statistical analysis}

For the kallikrein activity assay, two-way ANOVA was used and $P$-values were considered significant at $<0.05$. Statistical analyses were performed using GraphPad Prism 5 (La Jolla, San Diego, CA, USA).

\section{Results}

Changes in the mouse plasma proteome in the presence of schistosomes

As assessed by 2D-DIGE, normal mouse plasma in which adult schistosomes were incubated for $1 \mathrm{~h}$ at $37{ }^{\circ} \mathrm{C}$ 
displays a different proteomic profile compared with plasma incubated without schistosomes. The 2D electrophoresis pattern of both plasma samples is shown in Fig. 1a (right) where spots coloured red indicate proteins that are in greater relative abundance in the plasma sample that contained worms while spots coloured green are in greater abundance in the control plasma sample (without worms). The vast majority of proteins display no detectable difference between the two samples and these appear yellow.

In this work, we focus on the most striking differences between the protein profiles of the two plasma samples, highlighted in the boxed area in Fig. 1a. This area is enlarged in the left panel of Fig. 1a and contains four spots (numbered 1-4). Mass spectrometry analysis, following recovery and trypsin digestion of these protein spots, reveals that all are high molecular weight kininogen (HK, also known as kininogen-1; accession designation: KNG1_MOUSE) or fragments thereof. Examination using ImageQuant software reveals that spots 1 and 2 are found in greater relative abundance in the control plasma sample (4.6-fold and 2.1-fold, respectively) and appear green, while red spots 3 and 4 are found in greater relative abundance in the sample that contained worms (3.3-fold and 3.1-fold, respectively). Figure 1b, left panel, depicts an analysis of gel spot 2 using the DeCyder software in which the sizable protein peak (bounded by a yellow line in the figure) that is seen in the control sample ("- parasites") is greatly diminished in the sample containing parasites ("+ parasites"). DeCyder analysis of spot 4 (Fig. 1b, right panel) shows the
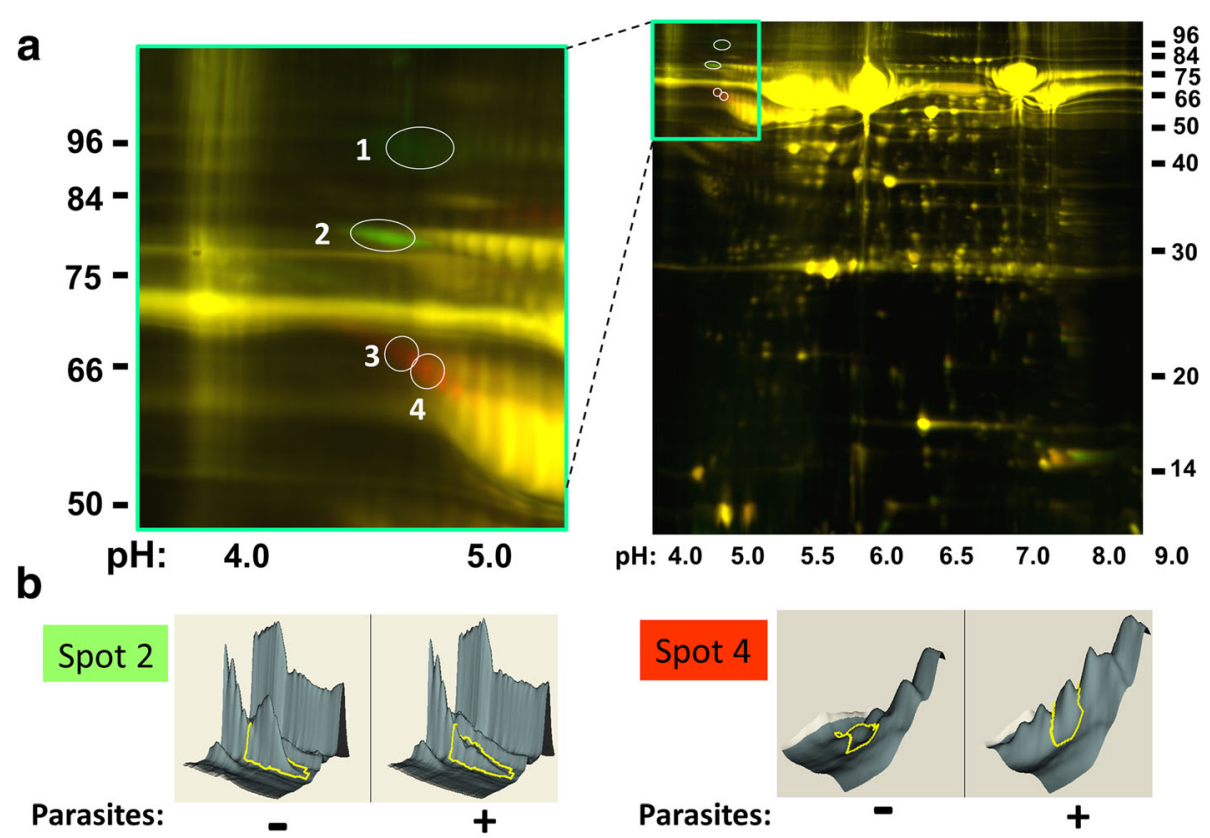

C

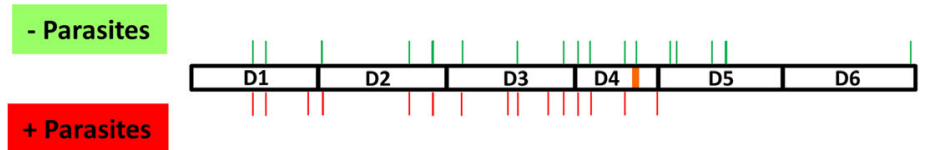

Fig. 1 Impact of schistosomes on the mouse plasma proteome. a Image (right) showing 2D gel resolution of samples obtained following normal heparinized mouse plasma incubation for $1 \mathrm{~h}$ at $37^{\circ} \mathrm{C}$ either in the presence of schistosome parasites (red) or without parasites (green). Most proteins are the same in both samples and appear yellow. The numbers on the sides of the images represent molecular mass markers ( $\mathrm{kDa}$ ) and the numbers at the bottom represent pH values. The section containing protein spots of interest is bounded by a green box at the top and this section is magnified and shown on the left. Four spots are circled and numbered. Two exhibits greater relative abundance in the absence of parasites (green spots 1, 2); two are more abundant in the presence of parasites (red spots 3 and 4). b Gel spot analysis by DeCyder software. The left panel depicts an analysis of gel spot 2. Here the sizable protein peak (bounded by a yellow line in the figure) that is seen in the control sample (- parasites) is greatly diminished in the sample containing parasites (+ parasites). Spot 4, right panel, shows the opposite effect in which the identified spot is barely detectable in the control sample (-) but becomes prominent in the presence of the worms (+). c Diagrammatic representation of the HK protein with its 6 domains (D1-D6). The 9-amino acid vasodilator bradykinin is contained within domain 4 and is indicated by an orange line. The positions of peptides that were identified by mass spectroscopy following trypsin digestion of 2D-DIGE spots 1 and 2 are indicated by thin green lines protruding above the HK protein. Positions of peptides identified by mass spectroscopy following trypsin digestion of 2D-DIGE spots 3 and 4 are indicated by red lines protruding below the protein 
opposite effect in that the identified spot is barely detectable in the control sample (left panel) but is strikingly revealed in the presence of the worms (right panel). Figure 1c depicts the HK protein with its 6 domains (D1-D6). The 9-amino acid vasodilator bradykinin is contained within domain 4 (D4) and is depicted in Fig. 1c by an orange line. The positions of peptides that were identified by mass spectroscopy following trypsin digestion of 2D-DIGE spots 1 and 2 are indicated by thin green lines protruding above the HK sequence in Fig. 1c. Peptides identified by mass spectroscopy following trypsin digestion of 2D-DIGE spots 3 and 4 are indicated by red lines protruding below the sequence. Additional file 1: Table S1 provides details regarding the peptides detected. Since no peptides derived from the carboxyl end of HK are detected from protein spots 3 and 4, this suggests that schistosomes are responsible for the generation of carboxyl-truncated forms of $\mathrm{HK}$. The data suggest cleavage of $\mathrm{HK}$ in the presence of the parasites beyond HK domain 4. This analysis does not provide clues as to the differences in the migration pattern of HK spot 1 vs spot 2 (presumably both are the full-length proteins), nor for differences between spot 3 vs spot 4 (truncated forms of $\mathrm{HK}$ ) although differential post-translational modification (e.g. glycosylation) may contribute. Other differences between the plasma samples, not related to $\mathrm{HK}$, will be explored in a separate publication.

\section{Analysis of HK cleavage by schistosomes}

To validate our proteomic findings regarding HK, we incubated living schistosomes with commercially obtained human HK (purified from plasma) and recovered samples $6 \mathrm{~h}$ and $24 \mathrm{~h}$ later. These samples were resolved by SDS-PAGE, blotted to a membrane and probed with the polyclonal anti-HK antibody. Results of this analysis are shown in Fig. 2a. At the $6 \mathrm{~h}$ time point it is clear that the presence of parasites (either schistosomula (S) or adult male worms (M)) does lead to HK cleavage: a $\sim 40 \mathrm{kDa}$ band is apparent (top arrow, Fig. 2a) that is not seen in the control sample incubated without worms ("-" lane). Prolonged incubation (to $24 \mathrm{~h}$ ) results in an accumulation of the $\sim 40 \mathrm{kDa}$ band (most apparent in the $24 \mathrm{~h}$ schistosomula (S) lane) and the appearance of a second cleavage product at $\sim 16 \mathrm{kDa}$ (bottom arrow, Fig. 2a). Neither is seen in the control sample at $6 \mathrm{~h}$ or $24 \mathrm{~h}$. The appearance of these bands is coincident with the diminution in the intensity of the full-length HK protein running at $120 \mathrm{kDa}$.

Next, HK was incubated with commercially obtained human kallikrein. Samples recovered $6 \mathrm{~h}$ and $24 \mathrm{~h}$ after incubation were resolved by SDS-PAGE, blotted to a membrane and probed with the polyclonal anti-HK antibody. The pattern of HK cleavage by kallikrein at the $24 \mathrm{~h}$ time point is compared to that generated by schistosomes in Fig. 2b (the $6 \mathrm{~h}$ and $24 \mathrm{~h}$ samples yielded identical patterns). As expected, kallikrein cleaves HK to generate two major products, a light chain and a heavy chain, as shown in Fig. 2b, left lane. This pattern is very different from that seen following schistosome incubation with HK (Fig. 2b, central lane) where the characteristic 40 $\mathrm{kDa}$ and $16 \mathrm{kDa}$ bands are again revealed. A control sample (HK alone) is also shown in Fig. 2b, right lane.

To explore what kind of schistosome protease is involved in substrate cleavage, parasites were incubated with $\mathrm{HK}$ in the presence of the serine protease inhibitor PMSF $(0.5 \mathrm{mM})$ or the presence of the cysteine protease inhibitor E64c $(0.1 \mathrm{mM})$ and the results are shown in Fig. 2c. It is clear that adding PMSF does not noticeably impede the ability of schistosomes to cleave HK (Fig. 2c, left panel). The characteristic $40 \mathrm{kDa}$ cleavage fragment (arrow) is seen both in the presence (Fig. 2c, lane $+/+$ ) and the absence (lane $+/-$ ) of inhibitor. In contrast, adding E64c does block HK cleavage (right panel, lane $+/+$ ); the HK profile seen in the presence of E64c is essentially the same as that seen in the control lane (HK alone, lane -/-). Note that following incubation of worms with these inhibitors, no obvious morphological differences were seen between them and control, untreated worms.

\section{Kallikrein activity assay}

The plasma serine protease kallikrein is known to cleave HK to generate the vasodilator bradykinin $[18,19]$. Since we have shown here that schistosomes do target HK for cleavage, the question arose as to whether the worms possess kallikrein (or kallikrein-like) activity. To test this, live worms were incubated with a chromogenic kallikrein substrate (D-Pro-Phe-Arg-pNa) [13] whose cleavage was monitored at $\mathrm{OD}_{405}$. Figure 3 shows the result of this assay. No convincing cleavage of the product was detected in the presence of the live parasites. Parasites incubated with substrate beyond 1 hour (and tested at 2, 4, 8, 12 and $24 \mathrm{~h}$ ) similarly failed to generate a detectable product. In contrast, adding human kallikrein to the assay results in clear and rapid cleavage of the substrate (Fig. 3). Adding the serine protease inhibitor PMSF to the assay impedes the ability of kallikrein to cleave this substrate, as expected.

\section{Bradykinin detection}

While the work presented here shows that schistosomes can cleave $\mathrm{HK}$, there is no evidence that they possess kallikrein activity (which would ordinarily release bradykinin from HK). Of course, it remains possible that the worms possess a non-kallikrein activity that nonetheless yields bradykinin. To assess whether the parasites can generate bradykinin as a product of HK cleavage, we employed a competitive bradykinin ELISA assay.

Here, human HK was incubated with living schistosomes (5 males) or commercially obtained human kallikrein $(1 \mu \mathrm{g})$ and samples were recovered $6 \mathrm{~h}$ and $24 \mathrm{~h}$ later. Under these conditions, $\mathrm{HK}$ is cleaved by both 


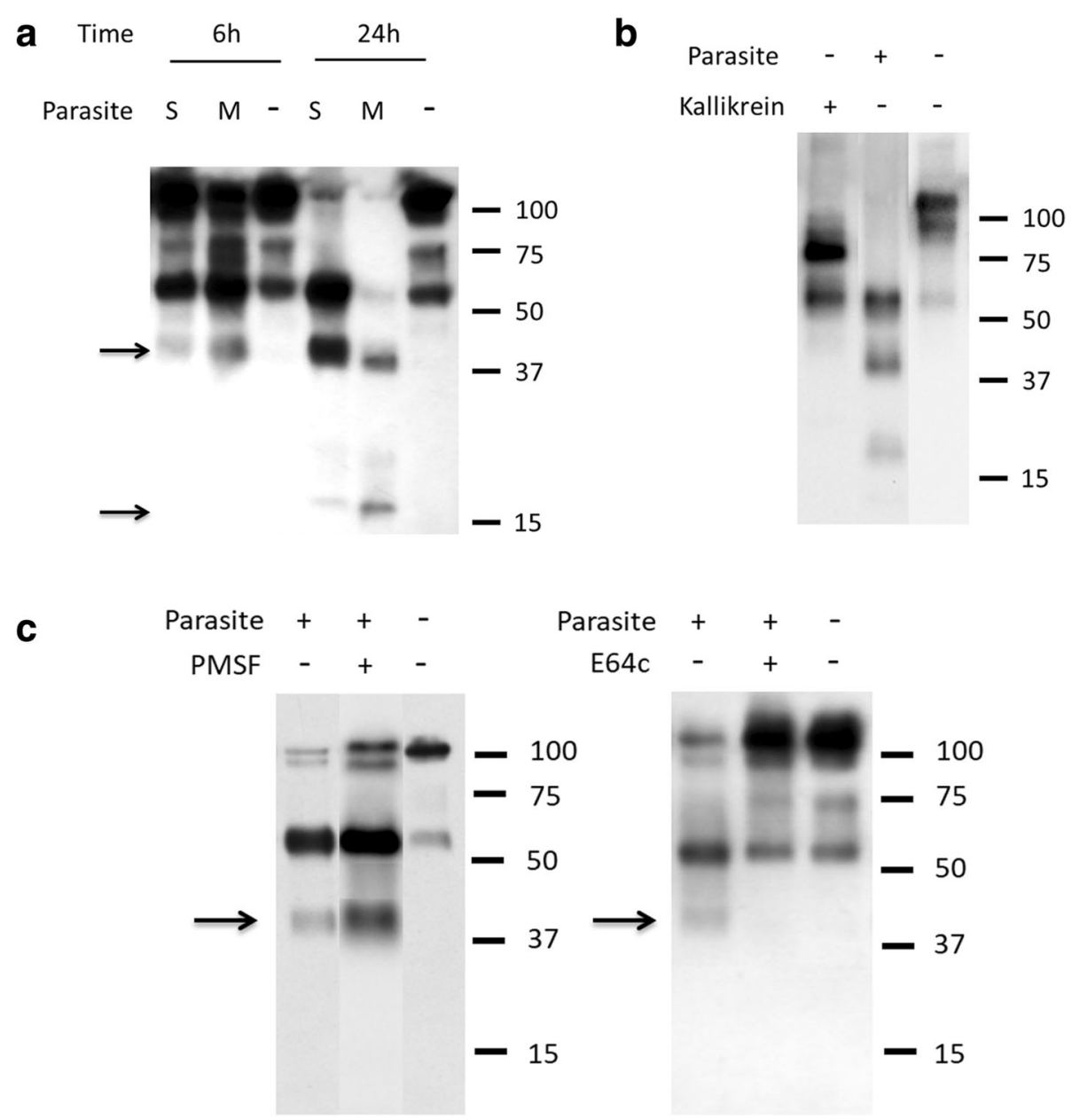

Fig. 2 Analysis of HK cleavage by schistosomes using western blotting. a High molecular weight kininogen (HK) was incubated in the absence $(-)$ or presence of parasites ( $S$, schistosomula; $M$, males) for different time periods ( 6 or 24 h, as indicated). Samples were resolved by SDS-PAGE, blotted to membrane and probed by western blotting. A number of prominent HK degradation products are detected (at $\sim 40$ and $16 \mathrm{kDa}$, arrows) only in the presence of parasites. The numbers on the right indicate molecular mass markers (kDa). $\mathbf{b}$ Parasites or kallikrein, as indicated, were incubated with HK for $24 \mathrm{~h}$ and cleavage products were resolved by SDS-PAGE, blotted to membrane and probed for HK digestion by western blotting. Numbers represent molecular mass markers ( $\mathrm{kDa}$, right). $\mathbf{c ~ H K}$ was incubated for $6 \mathrm{~h}$ with parasites either in the presence or absence of serine protease inhibitor PMSF $(0.5 \mathrm{mM})$, as indicated (left panel); or in the presence or absence of cysteine protease inhibitor E64c (0.1 mM) (right panel). The presence of PMSF (left panel) does not impede parasite-mediated cleavage of HK. The characteristic $\sim 40 \mathrm{kDa}$ product is indicated by the arrow. In contrast, the presence of E64C (right panel) does impede parasite-mediated cleavage of HK

schistosomes and kallikrein (as shown by western blot analysis, Fig. 2, described above). Samples were then diluted, added in replicate to a bradykinin ELISA plate to monitor bradykinin generation, and processed as described in methods. The $6 \mathrm{~h}$ and $24 \mathrm{~h}$ results are essentially the same and Fig. 4 shows the results of this analysis for the $24 \mathrm{~h}$ time point. It is clear that while, as expected, kallikrein generated easily detectable bradykinin (HK + Kallikrein), adult male schistosomes did not (HK + Males); no schistosome-cleaved HK sample yielded detectable bradykinin. The values obtained for the "schistosome + HK" samples were indistinguishable from those obtained using either $\mathrm{HK}$ alone or the zerobradykinin control sample (ANOVA: $F_{(2,6)}=4.07, P=0.14$ ).
The positive (+) and negative (-) controls are a bradykinin standard $(2.8 \mathrm{nM})$ and no bradykinin, respectively.

\section{Discussion}

In this study, we examined the impact of schistosomes on the murine plasma proteome, to gain insight into how the parasites interact with these host vascular components. It is clear that even in a short period (one hour) schistosomes can change the composition of plasma quite considerably. Using 2D-DIGE, we identified several protein spots that were markedly different in plasma that contained worms compared to plasma without worms. The biggest difference concerns the blood protein high molecular weight kininogen (HK). Four prominent spots contain 


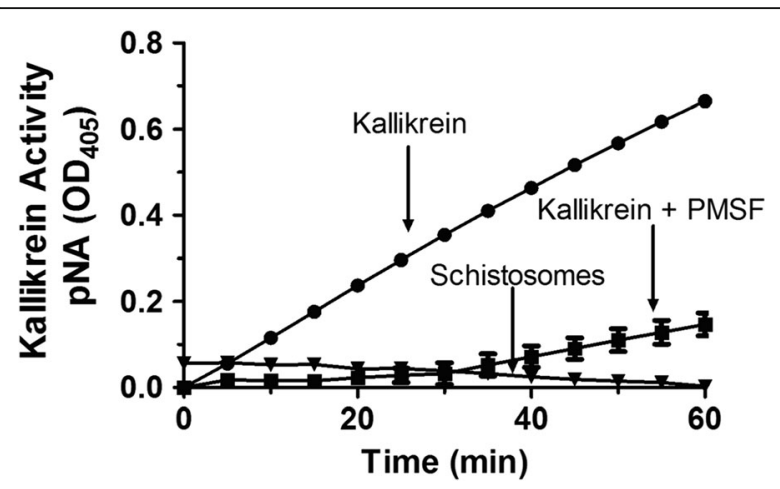

Fig. 3 Kallikrein activity assay. Cleavage of kallikrein substrate (H-D-Pro-Phe-Arg-pNa) by living schistosomes (triangle) and human kallikrein $(0.02 \mu \mathrm{g})$ in the presence (square) or absence (circle) of PMSF (2 mM) (Mean +/- SD, $n=3$ ). Released pNa was measured every 5 minutes at $\mathrm{OD}_{405}$. All conditions differ significantly from "Kallikrein"; two-way ANOVA: $F_{(24,52)}=94.76, F_{(2,52)}=2318.22$, $F_{(12,52)}=124.82, P<0.0001$

this protein or fragments thereof. This paper focuses exclusively on the impact of schistosomes on HK. Other changes to the murine proteome will be published elsewhere.

Two forms of HK are found in greater abundance in control plasma (identified as spots 1 and 2) and two forms are found in greater abundance in plasma that contained worms (spots 3 and 4). The peptides identified following trypsin digestion of recovered spots 1 and 2 span all domains of the protein. In contrast, peptides identified following trypsin digestion of recovered spots 3 and 4 span HK domains 1-4; none are derived from domains 5 or 6 . This suggests that protein spot 3 and 4 are both carboxyl truncated forms of full-length HK (spots 1 and 2).

To confirm that schistosomes were capable of cleaving HK (and not just in the context of murine plasma),

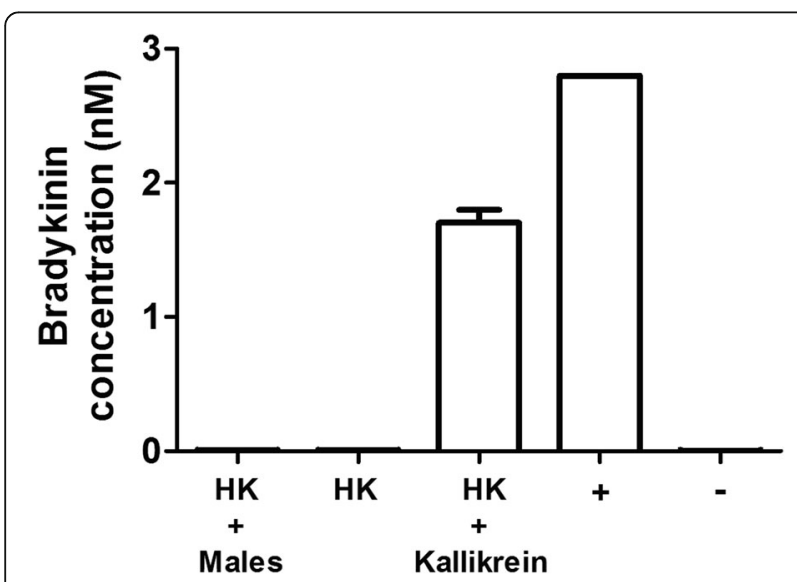

Fig. 4 Bradykinin detection. Bradykinin generated following incubation of HK with either male schistosomes (HK + males) or with pure human kallikrein (HK + Kallikrein) or alone (HK) for $24 \mathrm{~h}$ at $37^{\circ} \mathrm{C}$. Positive (+) and negative (-) controls used in this ELISA assay are bradykinin, $2.8 \mathrm{nM}$ and $0 \mathrm{nM}$. (Mean $+/-\mathrm{SD}, n=3$ ) worms were first incubated in buffer containing commercially obtained HK. Next, samples were recovered, resolved by SDS-PAGE, blotted to a membrane and probed with polyclonal anti-HK antibodies. This analysis confirms that the parasites are indeed capable of cleaving HK. Most prominent is the appearance of a $40 \mathrm{kDa}$ fragment and, following prolonged incubation, the appearance of a $16 \mathrm{kDa}$ fragment. Both intravascular larval stage parasites (schistosomula) and adult male worms are capable of cleaving HK to generate this characteristic digestion pattern. This pattern is distinct from that generated following cleavage of $\mathrm{HK}$ by the host enzyme kallikrein, which generates two characteristic major fragments (as well as the 9-amino acid peptide bradykinin).

While our finding is that parasite cleavage of HK is unlike that of host kallikrein cleavage of HK, it has been reported that S. mansoni homogenates do possess a kallikrein-like activity; this is associated with an uncharacterized $66 \mathrm{kDa}$ protein, sK1, that has been described as hydrolyzing the kallikrein synthetic substrate d-ProPhe-Arg-p-nitroanilide [13]. Also, an S. mansoni cDNA encoding a $\sim 35 \mathrm{kDa}$ murine kallikrein homolog was identified, SmSP1 [14]. Localization of sK1 and SmSP1 suggest that they might be host-interactive and could, therefore, endow schistosomes with a kallikrein-like activity in vivo. Thus, in this work we set out to determine if intact, living schistosomes could be directly demonstrated to exhibit kallikrein activity by incubating live worms with the kallikrein substrate noted above. From this work, we find no evidence that live worms cleave this reagent. As a positive control, kallikrein, as expected, gives rise to rapid and sustained substrate cleavage and this activity can be inhibited by the addition of the serine protease inhibitor, PMSF. We, therefore, hypothesize that the substrate-cleaving sK1 schistosome protein is not host exposed and therefore plays no role in parasite interaction with HK.

As noted earlier, HK is the source of the 9-amino acid vasodilatory peptide bradykinin and kallikrein-mediated cleavage of HK releases bradykinin [12, 20]. We have shown above that schistosomes can cleave HK, yet they do not possess kallikrein activity. We are interested in learning whether the worms nonetheless retain the ability to generate bradykinin (in a non-kallikrein manner). We argue that being able to generate bradykinin might benefit the worms because of its vasodilatory properties; it could help the parasites control blood movement around them. Bradykinin can additionally act on the vascular endothelium to induce the production of the prostaglandin $\mathrm{PGI}_{2}$ [11]. $\mathrm{PGI}_{2}$ is an inhibitor of platelet activation, an activity that would also assist schistosomes. On the other hand, neutrophil, as well as mast cell, activation has been functionally linked to bradykinin production, which may be damaging for the parasites [21]. In this work, we directly measured bradykinin production following 
parasite-mediated cleavage of HK. No bradykinin was detected after parasite treatment. In contrast, kallikreinmediated cleavage of $\mathrm{HK}$ yields easily measurable levels of bradykinin. This work confirms the observation that living schistosomes do not possess kallikrein activity; while the worms can cleave HK, they do not generate detectable levels of bradykinin. Whether further cleavage of the schistosome-generated HK fragments by host proteases leads to the eventual release of bradykinin is not known. One caveat arises from the report that an $S$. mansoni prolyl oligopeptidase (SmPOP) can degrade bradykinin [22]. This protein is found in the adult worm parenchyma and the tegument but no tegumental proteomic study finds it at the host-parasite interface [23-26]. Nonetheless, live adult worms are reported to cleave bradykinin suggesting that SmPOP on worms can access the peptide [22]. These data leave open the unlikely possibility that schistosomes can generate bradykinin, but it is rapidly broken down via SmPOP.

One reason that schistosomes cleave HK may be to disrupt its normal function in the initiation of blood coagulation. Figure 5 highlights the involvement of HK in several aspects of host metabolism. For example, HK acts as a cofactor in the conversion of factor XII to its active form (factor XIIa). Activated factor XIIa converts prekallikrein to kallikrein which then activates more factor XII. HK is an important cofactor in both reactions. HK is also necessary for the activation of factor XI by factor XIIa [11]. By cleaving a vital co-factor (HK) in the initiation of blood coagulation, the worms may impede blood clot formation around them in vivo. Certainly, worms within blood vessels do not seem perturbed by thromboses $[20,27]$ and ex vivo they can severely impede the ability of blood to clot [28]. However, whether HK cleavage by itself would necessarily impede coagulation remains to be determined; kallikrein cleavage of $\mathrm{HK}$ is reported to enhance its coagulant activity [29]. A lot depends on precisely where schistosomes cleave the HK molecule to destroy, or expose, specific domains. HK domain 3, in recombinant form, can inhibit thrombin-induced platelet aggregation [29]; domain 5 too has been identified as a potent inhibitor of platelet aggregation [29]. We hypothesize that generating fragments containing these domains may also benefit intravascular worms by further obstructing coagulation.

Impeding the activation of factor XII (by schistosome mediated HK cleavage) may have benefits for the worms beyond an impact on coagulation. For instance, since factor XIIa stimulates neutrophil aggregation [30], interleukin-1 expression in monocytes [31], and initiates the classical complement cascade [32], the ability of the worms to block its formation may additionally minimize the concentration of inflammatory mediators in their vicinity.

The question remains as to how schistosomes cleave HK to generate the characteristic pattern of protein fragments. Since the host HK cleaving enzyme kallikrein is a serine protease, we first set out to determine if the worms also possessed surface serine proteases that might be responsible for HK cleavage. To test this, parasites were incubated with HK plus PMSF (a serine protease inhibitor) but this did not impede the worm's ability to cleave HK. In contrast, when the parasites are incubated with the cysteine protease inhibitor E64c, their HK cleaving ability was severely impaired. This result provides strong evidence for the view that schistosome cysteine proteases are responsible for HK cleavage. This result is also consistent with tegumental proteomic work which places cysteine proteases belonging to the calpain family in the parasite surface membranes but no serine proteases there [24, 25]. Calpains are calcium-dependent cysteine proteases that engage in limited cleavage of their target proteins [33-35].

We have cloned the two calpain proteases identified by proteomic analysis as being expressed in the $S$. mansoni tegument. These are designated SmCalp1 and SmCalp2 and they display a highly conserved classical calpain domain organization [36]. Both are highly expressed in the parasite's intravascular life forms. Immunolocalization and activity-based protein profiling experiments confirm their presence at the host-parasite interface [36]. Furthermore, we have shown that living parasites exhibit surface calpain activity [36]. While we show here that live worms do not

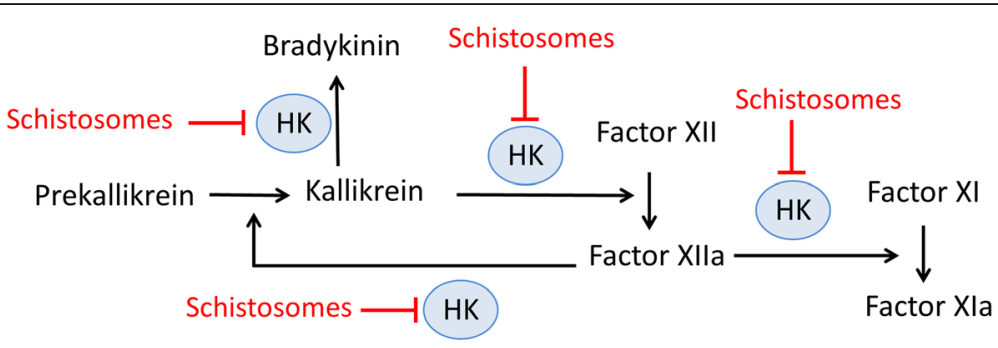

Fig. 5 Depiction of biochemical pathways involving high molecular weight kininogen (HK, blue circles) that schistosomes may disrupt (red). HK is a cofactor in the conversion of coagulation factor XII to its active form (Factor XIla) and the conversion factor XI to its active form (Factor Xla). HK is also involved in the Factor Xlla cleavage of prekallikrein to its active form, kallikrein. Finally, HK is cleaved by kallikrein to generate bradykinin. Cleavage of HK by parasites has the potential to disrupt all of these pathways 
cleave a kallikrein substrate (D-Pro-Phe-Arg-pNa), earlier work shows that living worms can cleave a calpain substrate (DABCYL-Thr-Pro-Leu-Lys-Ser-Pro-Pro-ProSer-Pro-Arg-EDANS) [36]. Cleavage of the latter is blocked in the absence of calcium and the presence of the calpain inhibitor E64c [36]. While calpains are invariably reported to be exclusively intracellular (except in diseased or injured tissues), it appears that schistosomes display unique, constitutive, functional extracellular calpain activity. In this work, we show that schistosomes can cleave the blood clotting protein HK in a manner that is inhibitable by the non-cell permeable, calpain inhibitor E64c. Since domains 2 and 3 of $\mathrm{HK}$ are designated as being involved in "cysteine protease inhibition" [37, 38], it is ironic that schistosome cysteine proteases may cleave this protein.

Other pathogens have been shown to interact with HK (e.g. the fungus Candida spp. [39, 40]) and some possess proteases that cleave HK (e.g. the bacteria Streptococcus pyogenes [41] and Porphyromonas gingivalis [42]). Our work is the first to show that a metazoan parasite $(S$. mansoni) can also cleave $\mathrm{HK}$ and our data differ from that reported for the bacterial pathogens in that no bradykinin is generated following worm-mediated HK cleavage.

In previous work, we showed that schistosomes are also capable of cleaving another host blood clotting protein, fibronectin, and that this activity can also be inhibited by E64c [36]. We hypothesize that the cysteine proteases SmCalp1 and/or SmCalp2 cleave both fibronectin and HK and towards the same end, to help impede blood clot formation around the worms in vivo and to diminish the concentration of inflammatory mediators near them. Schistosomes possess other molecular mechanisms that have been hypothesized to help prevent local thromboses [20]. These include their possession of ectoenzymes that can cleave pro-thrombotic nucleotides like ADP [8] as well as surface molecules that can promote the generation of active plasmin, a major clot-degrading enzyme [9]. These combined capabilities would promote the ease of unrestricted movement of the parasites within the vasculature of their hosts.

\section{Conclusions}

We have shown in this study that even with a short period of time schistosomes can clearly have an impact on the murine plasma proteome, specifically on high molecular weight kininogen. With our previous work on identification of two cysteine proteases belonging to the calpain family (SmCalp1 and SmCalp2) on the surface of schistosomes, we show and conclude that they are likely responsible for the HK cleavage reported here. The cleavage of $\mathrm{HK}$ is probably another factor how schistosomes impede blood clotting and inflammation around them in vivo and so promote their ease of movement within the vasculature of their hosts.

\section{Additional file}

Additional file 1: Table S1. Sequences of peptides identified by mass spectrometry from protein spots 1-4 (as shown in Fig. 1a). Spots 1 and 2 are from plasma sample obtained in the absence of parasites (green) and spots 3 and 4 are from plasma sample that contained adult schistosomes (red), as described in methods. An " $x$ " indicates that the peptide was found in the indicated protein spot. The amino acid start and end positions of each peptide within the murine high molecular weight kininogen (accession designation: KNG1_MOUSE) sequence are given. (PDF $418 \mathrm{~kb}$ )

\section{Abbreviations}

HK: High molecular weight kininogen; NIH-NIAID: National Institutes of Health-National Institute of Allergy and Infectious Diseases; BRI: Biomedical Research Institute; SmSP1: Schistosoma mansoni serine protease 1; DMEM/ F12: Dulbecco's Modified Eagle Medium/Nutrient Mixture F-12; IACUC: Institutional Animal Care and Use Committees; 2D-DIGE: Two-dimensional differential in-gel electrophoresis; SDS-PAGE: Sodium dodecyl sulfate polyacrylamide gel electrophoresis; MS: Mass spectrometry; HBSS: Hanks balanced salt solution; PMSF: Phenylmethylsulfonyl fluoride; TMB: Tetramethylbenzidine;

SmPOP: Schistosome mansoni prolyl oligopeptidase

\section{Acknowledgements}

Infected snails were provided by Biomedical Research Institute (BRI) via the NIAID Schistosomiasis Resource Centre under NIH-NIAID Contract No. HHSN272201000005I. The funders had no role in study design, data collection and interpretation, or the decision to submit the work for publication.

\section{Funding}

This work was funded with support from National Institutes of Health-National Institute of Allergy and Infectious Diseases (NIH-NIAID) grant Al056273 to P.J. Skelly. The funders had no role in study design, data collection and interpretation, or the decision to submit the work for publication.

\section{Availability of data and materials}

The data sets supporting the conclusions of this article are included in the article and its additional file.

\section{Authors' contributions}

QW, ADD and PJS designed the experiments. QW and ADD conducted the experiments. QW, ADD and PJS wrote the manuscript. All authors read and approved the final manuscript.

\section{Ethics approval and consent to participate}

All protocols involving animals were approved by the Institutional Animal Care and Use Committees (IACUC) of Tufts University under protocol G2015-113. The study did not involve human subjects.

\section{Consent for publication}

Not applicable.

\section{Competing interests}

The authors declare that they have no competing interests.

\section{Publisher's Note}

Springer Nature remains neutral with regard to jurisdictional claims in published maps and institutional affiliations.

Received: 27 November 2017 Accepted: 8 February 2018

Published online: 14 March 2018

\section{References}

1. Colley DG, Bustinduy AL, Secor WE, King CH. Human schistosomiasis. Lancet. 2014;383:2253-64.

2. Steinmann P, Keiser J, Bos R, Tanner M, Utzinger J. Schistosomiasis and water resources development: systematic review, meta-analysis, and estimates of people at risk. Lancet Infect Dis. 2006;6:411-25. 
3. King $\mathrm{CH}$, Dickman K, Tisch DJ. Reassessment of the cost of chronic helminthic infection: a meta-analysis of disability-related outcomes in endemic schistosomiasis. Lancet. 2005;365:1561-9.

4. King $\mathrm{CH}$, Dangerfield-Cha M. The unacknowledged impact of chronic schistosomiasis. Chronic IIIn. 2008;4:65-79.

5. World Health Organization. Schistosomiasis Fact Sheet. http://www.who.int/ mediacentre/factsheets/fs115/en/. Accessed 1 Oct 2017.

6. Gryseels B, Polman K, Clerinx J, Kestens L. Human schistosomiasis. Lancet. 2006;368:1106-18.

7. Bhardwaj R, Skelly PJ. Purinergic signaling and immune modulation at the schistosome surface? Trends Parasitol. 2009;25:256-60.

8. Da'dara AA, Bhardwaj R, Ali YB, Skelly PJ. Schistosome tegumental ecto-apyrase (SmATPDase1) degrades exogenous pro-inflammatory and pro-thrombotic nucleotides. PeerJ. 2014;2:e316.

9. Figueiredo BC, Da'dara AA, Oliveira SC, Skelly PJ. Schistosomes enhance plasminogen activation: the role of tegumental enolase. PLoS Pathog. 2015;11:e1005335

10. Bhardwaj R, Skelly PJ. Characterization of schistosome tegumental alkaline phosphatase (SmAP). PLoS Negl Trop Dis. 2011;5:e1011.

11. Schmaier $\mathrm{AH}$. The contact activation and kallikrein/kinin systems: pathophysiologic and physiologic activities. J Thromb Haemost. 2016;14:28-39.

12. Lin L, Wu M, Zhao J. The initiation and effects of plasma contact activation: an overview. Int J Hematol. 2017;105:235-43.

13. Carvalho WS, Lopes CT, Juliano L, Coelho PM, Cunha-Melo JR, Beraldo WT, Pesquero JL. Purification and partial characterization of kininogenase activity from Schistosoma mansoni adult worms. Parasitology. 1998;117(4):311-9.

14. Cocude C, Pierrot C, Cetre C, Fontaine J, Godin C, Capron A, Khalife J. Identification of a developmentally regulated Schistosoma mansoni serine protease homologous to mouse plasma kallikrein and human factor I. Parasitology. 1999;118(4):389-96.

15. Da'dara AA, Skelly PJ. Gene suppression in schistosomes using RNAi. Methods Mol Biol. 2015;1201:143-64.

16. Milligan JN, Jolly ER. Cercarial transformation and in vitro cultivation of Schistosoma mansoni schistosomules. J Vis Exp. 2011;54:3191.

17. Laemmli UK. Cleavage of structural proteins during the assembly of the head of bacteriophage T4. Nature. 1970;227:680-5.

18. Schmaier AH. A novel antithrombotic mechanism mediated by the receptors of the kallikrein/kinin and renin-angiotensin systems. Front Med (Lausanne). 2016:3:61.

19. Ghebrehiwet B, Kaplan AP, Joseph K, Peerschke El. The complement and contact activation systems: partnership in pathogenesis beyond angioedema. Immunol Rev. 2016;274:281-9.

20. Mebius MM, van Genderen PJ, Urbanus RT, Tielens AG, de Groot PG, van Hellemond JJ. Interference with the host haemostatic system by schistosomes. PLoS Pathog. 2013;9:e1003781.

21. Hofman Z, de Maat S, Hack CE, Maas C. Bradykinin: inflammatory product of the coagulation system. Clin Rev Allergy Immunol. 2016;51:152-61.

22. Fajtova P, Stefanic S, Hradilek M, Dvorak J, Vondrasek J, Jilkova A, et al. Prolyl oligopeptidase from the blood fluke Schistosoma mansoni: from functional analysis to anti-schistosomal inhibitors. PLoS Negl Trop Dis. 2015;9:e0003827.

23. Sotillo J, Pearson M, Becker L, Mulvenna J, Loukas A. A quantitative proteomic analysis of the tegumental proteins from Schistosoma mansoni schistosomula reveals novel potential therapeutic targets. Int J Parasitol. 2015:45:505-16.

24. Castro-Borges W, Dowle A, Curwen RS, Thomas-Oates J, Wilson RA. Enzymatic shaving of the tegument surface of live schistosomes for proteomic analysis: a rational approach to select vaccine candidates. PLoS Negl Trop Dis. 2011;5:e993.

25. Braschi S, Curwen RS, Ashton PD, Verjovski-Almeida S, Wilson A. The tegument surface membranes of the human blood parasite Schistosoma mansoni: a proteomic analysis after differential extraction. Proteomics. 2006:6:1471-82.

26. Braschi S, Wilson RA. Proteins exposed at the adult schistosome surface revealed by biotinylation. Mol Cell Proteomics. 2006;5:347-56.

27. Tanabe M. Haemostatic abnormalities in hepatosplenic schistosomiasis mansoni. Parasitol Int. 2003:52:351-9.

28. Da'dara AA, de Laforcade AM, Skelly PJ. The impact of schistosomes and schistosomiasis on murine blood coagulation and fibrinolysis as determined by thromboelastography (TEG). J Thromb Thrombolysis. 2016;41:671-7.
29. Scott CF, Silver LD, Schapira M, Colman RW. Cleavage of human high molecular weight kininogen markedly enhances its coagulant activity. Evidence that this molecule exists as a procofactor. J Clin Invest. 1984;73:954-62

30. Wachtfogel YT, Pixley RA, Kucich U, Abrams W, Weinbaum G, Schapira M, Colman RW. Purified plasma factor Xlla aggregates human neutrophils and causes degranulation. Blood. 1986;67:1731-7.

31. Toossi Z, Sedor JR, Mettler MA, Everson B, Young T, Ratnoff OD. Induction of expression of monocyte interleukin 1 by Hageman factor (factor XII). Proc Natl Acad Sci USA. 1992;89:11969-72.

32. Ghebrehiwet B, Randazzo BP, Dunn JT, Silverberg M, Kaplan AP. Mechanisms of activation of the classical pathway of complement by Hageman factor fragment. J Clin Invest. 1983;71:1450-6.

33. Sorimachi $H$, Hata S, Ono Y. Calpain chronicle - an enzyme family under multidisciplinary characterization. Proc Jpn Acad Ser B Phys Biol Sci. 2011;87:287-327.

34. Zatz M, Starling A. Calpains and disease. N Engl J Med. 2005;352:2413-23.

35. Campbell RL, Davies PL. Structure-function relationships in calpains. Biochem J. 2012;447:335-51.

36. Wang Q, Da'dara AA, Skelly PJ. The human blood parasite Schistosoma mansoni expresses extracellular tegumental calpains that cleave the blood clotting protein fibronectin. Sci Rep. 2017:7:12912.

37. Pavord S, Kelton JG, Warner MN, Moore JC, Warkentin TE, Hayward CP. Proteolytic degradation of high molecular weight kininogen in acute thrombotic thrombocytopenic purpura. Br J Haematol. 1997;97:762-7.

38. Bradford HN, Jameson BA, Adam AA, Wassell RP, Colman RW. Contiguous binding and inhibitory sites on kininogens required for the inhibition of platelet calpain. J Biol Chem. 1993;268:26546-51.

39. Karkowska-Kuleta J, Zajac D, Bras G, Bochenska O, Seweryn K, Kedracka-Krok S, et al. Characterization of the interactions between human high-molecular-mass kininogen and cell wall proteins of pathogenic yeasts Candida tropicalis. Acta Biochim Pol. 2016;63:427-36.

40. Rapala-Kozik M, Karkowska J, Jacher A, Golda A, Barbasz A, Guevara-Lora I, Kozik A. Kininogen adsorption to the cell surface of Candida spp. Int Immunopharmacol. 2008:8:237-41.

41. Nitzsche R, Rosenheinrich M, Kreikemeyer B, Oehmcke-Hecht S. Streptococcus pyogenes triggers activation of the human contact system by streptokinase. Infect Immun. 2015;83:3035-42.

42. Scott CF, Whitaker EJ, Hammond BF, Colman RW. Purification and characterization of a potent 70-kDa thiol lysyl-proteinase (Lys-gingivain) from Porphyromonas gingivalis that cleaves kininogens and fibrinogen. J Biol Chem. 1993:268:7935-42.

\section{Submit your next manuscript to BioMed Central and we will help you at every step:}

- We accept pre-submission inquiries

- Our selector tool helps you to find the most relevant journal

- We provide round the clock customer support

- Convenient online submission

- Thorough peer review

- Inclusion in PubMed and all major indexing services

- Maximum visibility for your research

Submit your manuscript at www.biomedcentral.com/submit 